\title{
Electron Microscopy of Organic Solar Cells Thermally Stabilized with Fullerene Nucleating Agents
}

\author{
Stefan Gustafsson ${ }^{1}$, Olof Bäcke ${ }^{1}$, Samira Nik $^{1}$, Anke Sanz-Velasco ${ }^{2}$, Camilla Lindqvist ${ }^{3}$, Ergang Wang ${ }^{3}$, \\ Mats R. Andersson ${ }^{3}$, Christian Müller ${ }^{3}$ and Eva Olsson ${ }^{1}$ \\ ${ }^{1}$ Department of Applied Physics, Chalmers University of Technology, 41296 Göteborg, Sweden \\ ${ }^{2}$ Department of Microtechnology and Nanoscience - MC2, Chalmers University of Technology, 41296 \\ Göteborg, Sweden \\ ${ }^{3}$ Department of Chemical and Biological Engineering/Polymer Technology, Chalmers University of \\ Technology, 41296 Göteborg, Sweden
}

Organic Solar Cells (OSCs) have a great potential for solar energy harvesting due to their low material cost and cheap production. The most common and promising type of OSC is the bulk-heterojunction (BHJ) OSC, which have shown power-conversion efficiencies of 8-10\% [1]. The absorber layer in these OSCs consists of a mix of two phases; (i) a polymer, that serves as an electron donor, and (ii) a fullerene, that serves as electron acceptor. The performance of BHJ OSCs is directly related to the threedimensional microstructure of the absorber layer as the charge carriers generated must have access to paths of easy transport to avoid entrapment and recombination. It is thus necessary that the polymer and the fullerene phase form an intertwined continuous network on the nanoscale.

In order to upscale device fabrication and to meet requirements for solar cell standard test protocols the BHJ OSCs have to be able to withstand elevated temperatures of up to $140{ }^{\circ} \mathrm{C}$. This will, however, result in a coarsening of the nanostructure and thereby a degradation of their properties. In this work we have used transmission electron microscopy (TEM) to study how the three-dimensional BHJ OSC microstructure can be stabilized upon annealing at $130{ }^{\circ} \mathrm{C}$ using $\mathrm{C}_{60}$ as a fullerene nucleating agent. The photovoltaic blends comprise a non-crystalline thiophene-quinoxaline copolymer (TQ1) and the widely used fullerene derivative [6,6]-phenyl- $\mathrm{C}_{61}$-butyric acid methyl ester (PCBM). The addition of $\mathrm{C}_{60}$ ranges from 0 up to $20 \mathrm{wt} \%$ with respect to PCBM. Annealing was performed at $130{ }^{\circ} \mathrm{C}$ for $10 \mathrm{~min}$.

The TEM analysis was carried out using a Tecnai F20 equipped with a $\mathrm{LaB}_{6}$ electron gun and operating at $200 \mathrm{kV}$. Tomography tilt series were recorded between $\pm 60^{\circ}$ with a tilt angle increment of $1^{\circ}$; reconstructed using the FEI software Inspect $3 \mathrm{D}$ and visualized using the Amira software.

Figure 1 shows the resulting microstructure for blends with $0,5,10$ and $20 \mathrm{wt} \% \mathrm{C}_{60}$, respectively that have been annealed at $130{ }^{\circ} \mathrm{C}$ for $10 \mathrm{~min}$. Without additions of $\mathrm{C}_{60}$ a few micron-sized PCBM crystals are formed and a depletion area is formed around these crystals. This microstructure results in a deterioration of the device properties. Upon a gradual increase of the $\mathrm{C}_{60}$ addition a large number of smaller PCBM clusters of crystals are formed reaching an average size of around 200 nanometers and a separation distance less than $100 \mathrm{~nm}$ for $10 \mathrm{wt} \% \mathrm{C}_{60}$. The crystals also form a seemingly continuous network for $\mathrm{C}_{60}$ additions of more than $10 \mathrm{wt} \%$. Even upon annealing for more than 60 minutes the device characteristics are stable indicating that the microstructure do not coarsen further. Furthermore, from the images it is suggested that for additions of $\mathrm{C}_{60}$ up to $10 \mathrm{wt} \%$, the blends are only composed of two phases, i.e. fullerene crystals and a TQ1-rich phase suggesting that fullerene crystallization is to a large extent complete. However for the sample with $20 \mathrm{wt} \% \mathrm{C}_{60}$ there also seems to be an intermediate region connecting the crystals as seen in Figure 1. This may be an important contribution to the 
continuous network of fullerene species as tomography reconstructions of the $20 \mathrm{wt} \%$ sample show that the crystals themselves don't form a continuous network.

\section{References:}

[1] Z. He et al, Nature Photon 6 (2012), p. 591.
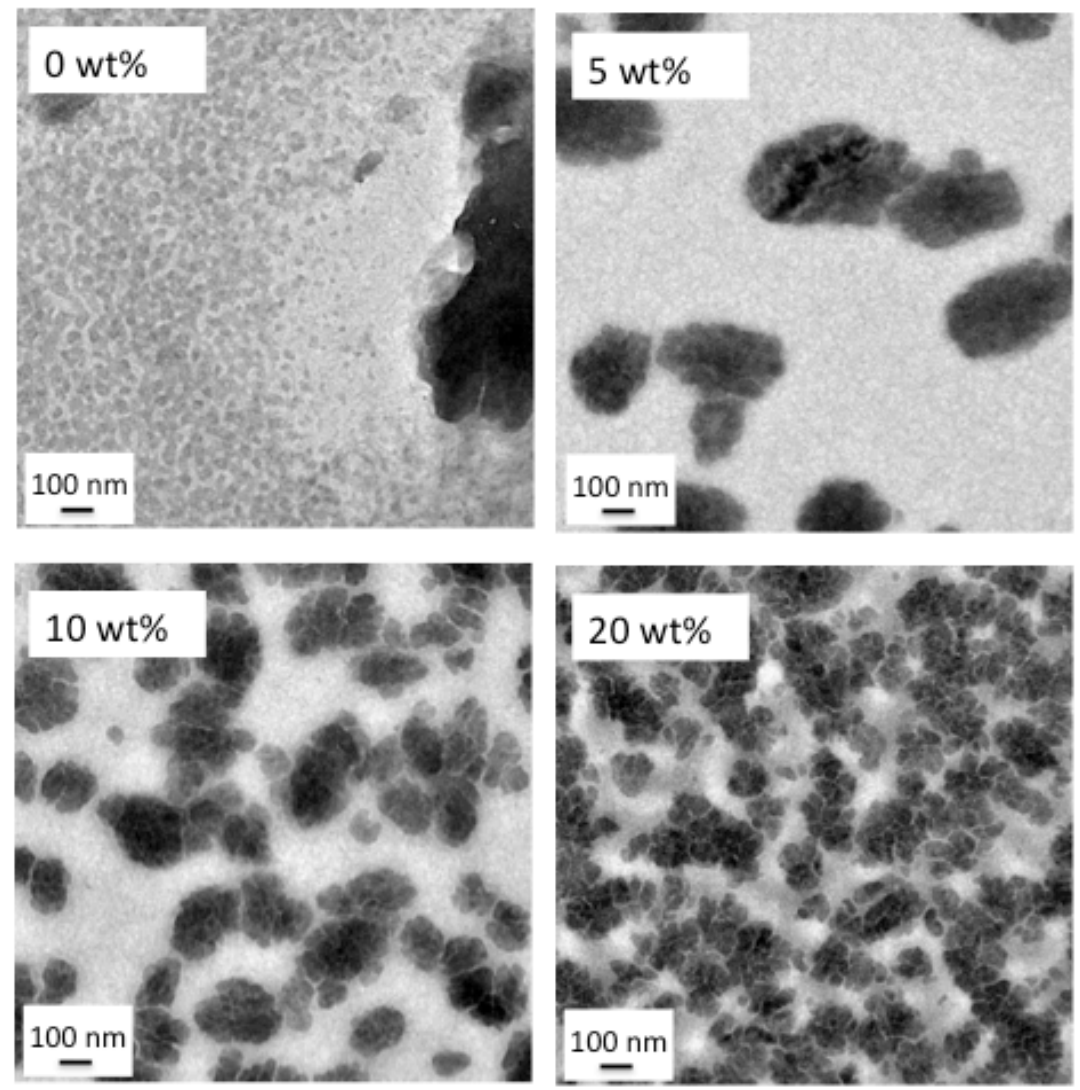

Figure 1. TEM images of TQ1:PCBM blends with additions of $0,5,10$ and $20 \mathrm{wt} \% \mathrm{C}_{60}$, respectively after annealing at $130{ }^{\circ} \mathrm{C}$ for $10 \mathrm{~min}$.

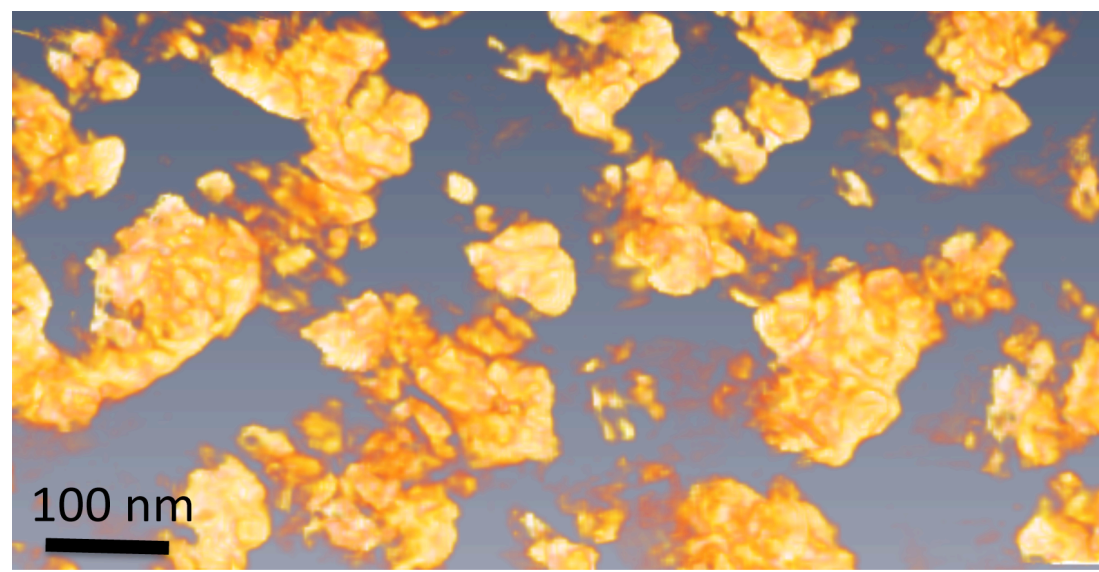

Figure 2. Reconstructed volume of the TQ1:PCBM sample with $20 \mathrm{wt} \% \mathrm{C}_{60}$. The crystals do not form a continuous network in this region. 